\title{
ON EDGE-DISJOINT CYCLES IN A GRAPH
}

\author{
J. W. Moon \\ (received March 21, 1964)
}

1. Introduction. Let $g(k)$ denote the least integer such that every graph $G(n, n+g(k))$, with $n$ vertices and $n+g(k)$ edges, contains at least $k$ edge-disjoint cycles; let $h(k)$ be similarly defined for planar graphs. Loops and multiple edges (i.e., cycles of length one and two) are permitted in both cases.

A trivial result is that $g(1)=h(1)=0$. Erdós and Pósa [2] showed that $g(2)=4$; Dirac and Erdös [1] showed that $h(2)=3$. In the present paper their arguments will be extended to show that

$$
\begin{aligned}
& g(3)=10, \\
& h(3)=7,
\end{aligned}
$$

and

$$
h(4)=11 \text {. }
$$

Further exact results would appear to require a stronger method of attack or the examination of a considerable number of special cases.

2. Proof that $g(3)=10$. We first show that every graph $G(n, n+10)$ contains at least three edge-disjoint cycles. This assertion is clearly true for $n=1$; suppose that it has been established for all $n<m$, where $m$ is some integer greater than one. Consider any graph $G=G(m, m+10)$. We may assume that $G$ has no cycle of length less than seven, for if it did the graph obtained by removing the edges of this cycle would still have at least $m+4$ edges and hence would contain

Canad. Math. Bull. vol. 7, no. 4, October 1964 
at least two edge-disjoint cycles since $g(2)=4$. So $G$ would contain at least three edge-disjoint cycles and there would be nothing to prove. If $\mathbf{x}$ is a vertex of degree less than two, then the induction hypothesis may be applied to the graph obtained from $G$ by removing $x$; if $x$ is of degree two then the induction hypothesis may be applied to the graph obtained from $G$ by removing $x$ and adding an edge joining the two vertices originally joined to $x$. From this it follows that we may assume that the degree of each vertex of $G$ is at least three. This implies that $m+10 \geqq \frac{3}{2} m$, or $m \leqq 20$. But it is easy to show (see the proof of Lemma 3 in [2]) that any graph each vertex of which has degree at least three and which contains no cycles of length less than seven must have at least 22 vertices. This contradiction suffices to establish the above assertion by induction. Therefore $g(3) \leqq 10$.

The graph in Figure 1 has 18 vertices, is regular of degree three, and its shortest cycles are of length six. Hence, if it had three edge-disjoint cycles they each would be of length six and each vertex would belong to one and only one of them.

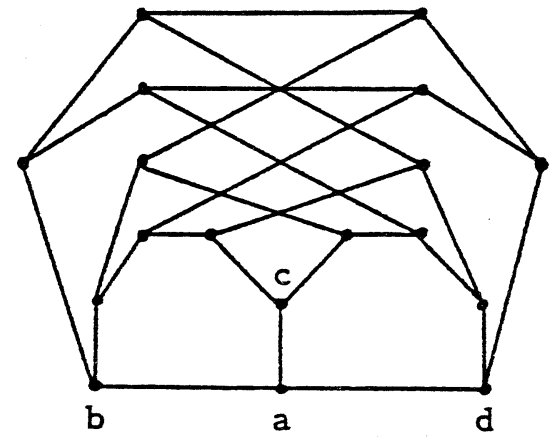

Figure 1

Consider the vertex $a$; it is easily verified that there are no cycles of length six passing through a via the edges $(b, a)$ and $(a, d)$. Hence, by symmetry, we may assume that if this graph contains three edge-disjoint cycles then the cycle passing through a contains the edges $(c, a)$ and $(a, d)$. There are only two cycles of length six with this property. But when the vertices of either of the se cycles are removed, along with their incident edges, the remaining graph consists of a hexagon 
with its three diagonals joining opposite vertices, some edges of which have been subdivided by the insertion of additional vertices. It is clear that such a graph does not contain two edge-disjoint cycles. From this it follows that the graph in Figure 1 does not contain three edge-disjoint cycles. Since it has 27 edges it must be that $g(3)>9$, and it is trivial to use this example to construct graphs $G(m, m+9)$ not containing three edge-disjoint cycles for any $m \geqq 18$. Combining this inequality with the earlier one, we have that $g(3)=10$.

3. A lemma. We sketch the proof of the following lemma, although it employs a rather familiar type of argument. (See Steinitz [4], pp. 7-8.)

LEMMA. If $G=G(n, e)$ is a planar graph such that the length of each cycle is at least five and the degree of each vertex is at least three, then $3 e \leqq 5 n-10$.

Proof. We may assume that $G$ is a planar map to which Euler's polyhedral formula may be applied; for, if it was not it could be made into one by adding appropriate edges, and it is not difficult to see that this could be done without forming any cycles of length less than five. Suppose that $G$ has a total of $f$ faces of which $f_{i}$ have $i$ sides. Then

$$
2 e=5 f_{5}+6 f_{6}+\ldots \geq 5\left(f_{5}+f_{6}+\ldots\right)=5 f \text {, }
$$

appealing to the hypothesis on the lengths of the cycles.

Substituting this inequality in Euler's formula, $n-e+f=2$, yields the required result.

4. Proof that $h(3)=7$. We first show that every planar graph $G(n, n+7)$ contains at least three edge-disjoint cycles. This assertion is certainly true for small values of $n$. Let $m$ be the smallest integer for which there exists a planar graph $G=G(m, m+7)$ for which it is not true. Using the fact that $h(2)=3$, the argument in $\S 2$ can be applied to show that the length of each cycle of $G$ is at least five and the degree of each vertex is at least three. Hence, $m+7 \geqq \frac{3}{2} m$ or $m \leqq 14$. But by the lemma, $3(m+7) \leqq 5 m-10$ or $m \geqq 15 \frac{1}{2}$. This contradiction establishes the assertion and shows that $h(3) \leqq 7$. 


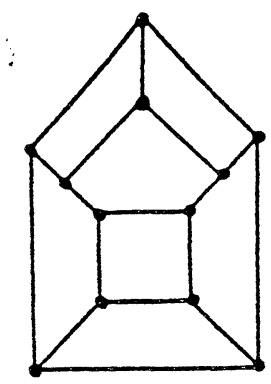

Figure 2

Consider the planar graph in Figure 2. If it contained three edge-disjoint cycles they would each have to be of length four. But it is clearly impossible to select three of the four cycles of length four without some edge being used more than once. This example, with 12 vertices and 18 edges, shows that $h(3)>6$ and such examples with $n$ vertices can be constructed for any $n \geqq 12$. Combining the two inequalities, we have that $h(3)=7$.

5. Proof that $h(4)=11$. It is easily verified that the graph of a regular dodecahedron, having 20 vertices and 30 edges, does not contain four edge-disjoint cycles. The examples obtained from this by subdividing its edges by inserting more vertices all show that $h(4)>10$.

We now show that every planar graph $G(n, n+11)$ contains at least four edge-disjoint cycles. Let $m$ be the smallest integer for which there exists a planar graph $G=G(m, m+11)$ without four such cycles. The same argument as used before shows that the length of each cycle of $\boldsymbol{G}$ is at least five and the degree of each vertex is at least three. Hence, $m+11 \geqq \frac{3}{2} m$ or $m \leqq 22$, and $3(m+11) \leqq 5 m-10$ or $m \geqq 21 \frac{1}{2}$, by the lemma. As $m$ is an integer it must be that $m=22$ and that the degree of each vertex of $G$ is exactly three.

A more careful analysis of the argument used in proving the lemma shows that if the planar graph $G$ has 22 vertices, is regular of degree three, and has no cycles of length less than or equal to four, then $G$ must be a planar map with 12 
pentagonal faces and one hexagonal face. But, as pointed out by Grünbaum and Motzkin [3], no such map exists.

This contradiction establishes the above assertion which when combined with the earlier inequality completes the proof that $h(4)=11$.

\section{REFERENCES}

1. G. Dirac and P. Erdons, On the maximal number of independent circuits in a graph, Acta Math. Acad. Sci. Hung. 14 (1963), 79-94.

2. P. Erdōs and L. Pósa, On the maximal number of disjoint circuits in a graph, Publ. Math. Debrecen 9 (1962), 3-12.

3. B. Grünbaum and T.S. Motzkin, The number of hexagons and the simplicity of geodesics on certain polyhedra, Canad. J. Math. 15 (1963), 744-751.

4. E. Steinitz, Vorlesungen úber die Theorie der Polyeder (Berlin, 1934).

\section{University College London}

